\title{
Analysis on Medical Insurance Payment Pattern between China and America
}

\author{
Wei WANG ${ }^{1}$, Wen-jing GONG ${ }^{1}$ and You-mei WANG ${ }^{2, ~ * ~}$ \\ ${ }^{1}$ School of Finance and Public Management, Harbin University of Commerce, Harbin \\ Heilongjiang, 150028 \\ ${ }^{2}$ Institute of Commercial Economics, Harbin University of Commerce, Harbin \\ Heilongjiang, 150028 \\ ${ }^{*}$ Corresponding author
}

Keywords: Medical insurance, Payment method, China, America.

\begin{abstract}
Medical insurance payment pattern plays a key role in the control of medical insurance expenses, and is also the key and difficult point in health insurance reform, so it is of great significance to do some research on this area. The paper introduces the medical insurance payment ways of China and America and their advantages and disadvantages, and finds out the advantages of American medical insurance payment methods, through comparing analysis in order to learn some experience. The purpose is to provide good suggestions for Chinese medical insurance reform, which includes carrying out the total prepaid amount, to adopt capitation payment method and to adopt commercial insurance as the leading medical insurance model, and to promote the development of China's medical insurance system.
\end{abstract}

\section{Introduction}

Medical insurance concerns people's well-being, the major issues of national economy and people's livelihood. Nowadays, some medical insurance problems appear in the economic boom in China, so does other countries in the international. Among them, the problem of medical insurance payment is particularly highlighted, mainly embodied in the rapid growth of the medical insurance expenses. This has became a huge burden for national finance. There are many reasons that cause medical costs soaring, such as the progress of science and medical technology. These all make the big increase in medical costs. With the increase of population aging, more and more old people are in need of medical services. The improvement of living standards and higher health consciousness also causes the increase of medical expense.

In the face of such difficult problem of the world, all countries take the corresponding measures to cope with the rising of health care costs. Among them, Japanese use the points to fix a price for health care. As the patients are hospitalized, they only need to pay the registration fee and the part they must pay by themselves, and the hospital will provide the medical expense settlement list and notes every a month for the rest part to the medical insurance organization.[1] The reward for the doctors is strictly calculated according to the medical insurance payment point meter, which has 500 pages. This point meter concludes detailed specification on a variety of diagnosis and treatment projects, medicine scope, and check projects. It rules that one single point is 10 yen. Medical insurance organization is rather strict about the payment of medical insurance. It should be paid after the audit by the medical insurance fund organization and medical joint. In Germany, they take total prepaid on physician reimbursement system as a way to pay for the doctors. The disease 
insurance foundation and insurance physicians association negotiate annual total medical expenses on the basis of the total medical expenses of the previous year, combining with the change of some factors such as price, population. And the insurance physicians association bear all the responsibility of the outpatient (the association of physicians charged according to the service assigned to each physician). [2] However, the study on the pattern of medical insurance payment is few, especially, there are fewer researches written by the method of comparison, can be used as reference, that can provide reference for the pattern of Chinese medical insurance. The study starts with the present situation of Chinese and American medical insurance. Compare America with China from the angle of medical insurance payment pattern, hoping to learn good experience, finding the advantages of American payment pattern, and solving practical problems in China's medical insurance combining with China's national conditions.

\section{The Analysis of Current Situation on Medical Insurance payment pattern in China and America}

\section{Medical Insurance Payment Pattern in China}

At present, there are three types of medical insurance system mainly in China. The three types of systems are widely used and have the widest coverage. They are basic medical insurance for urban employees, basic medical insurance for urban residents and the new rural cooperative medical system. Among them, the urban basic medical insurance makes a great contribution to expand health insurance coverage in our country, as shown in table 1. And our country adopt fee for service as the medical insurance payment pattern. Chinese government has always adopted the payment by health service. This system is formulated according to the type and quantity of services items received by the patients, and medical service expenditure predetermined in the payment of unit price that is similar to piece rate system. [3] It is the most traditional and most widely used medical insurance payment pattern. The advantage is that it has a certain incentive effect, and can fully supervise the medical service providers to work more actively. Simultaneously, it promotes medical workers to improve the quality of medical services. And they even make a diagnosis and treatment for more patients with their breaking space. They are willing to make a diagnosis and treatment for the patients who need more and more complex services without any complaint. From the angle of the patients, this approach provides more choices and opportunities for them. Also, this approach receives a high degree of acceptance and satisfaction of the masses. The drawback is that the payment method charges in accordance with the number and types of services, so to obtain more revenue service providers may propose unnecessary high cost service projects to the sick, and even it comes out the social phenomenon of decomposition of project fees and other irregularities. Because of the market information asymmetry, patients can only choose medical service projects according to a doctor's proposal, resulting in high cost of getting medical treatment and the difficulty of getting medical service.

Table1. The Insured Population of Urban Basic Medical Insurance in China Unit: ten thousand people

\begin{tabular}{|c|c|c|c|}
\hline Year & Urban Residents & Urban Employees & Total \\
\hline 2010 & 29629.4 & 27443.1 & 57072.5 \\
\hline 2011 & 27155.7 & 26485.6 & 53641.3 \\
\hline 2012 & 22116.1 & 25227.1 & 47343.2 \\
\hline 2013 & 19528.3 & 23734.7 & 43263.0 \\
\hline 2014 & 31450.9 & 28296.0 & 59746.9 \\
\hline
\end{tabular}


Due to the payment by health service is not perfect, so since the beginning of the 20th century, Chinese have began to explore a new payment system of medical insurance actively-total prepaid amount. Total prepaid system is consulted together by medical treatment insurance institutions and hospitals. They predetermine the total annual budget. During the year, the hospital's medical costs will be paid from the total annual budget. The rest of the money belongs to the hospitals and the money of over budget will be paid by the hospitals themselves. Simple speaking, the total prepaid amount is to set up a top line for medical services within the prescribed medical year. Regardless of the cost of the supplier actually incurred, they treat the amount of the top line as boundary value to determine the medical expense of the medical institutions. The part beyond the top line shall be borne by the hospital, and the rest shall be owned to the hospital. This kind of medical insurance payment pattern is greatly arises under the background of the excessively rapid growth of medical expense at the historic moment. It has the advantage that the hospital's income will not increase as the growth of the service quantity. Thus, force medical institutions to control health care costs and reduce unnecessary spending and the waste of health resources actively. Medical insurance management agency has higher control power for health insurance fees. In this way, they can avoid the fund risk effectively.[4] Also it is helpful to arouse the consciousness of the medical service supplier on cost control, urge them to reduce service costs, improve the utilization of medical resources and promote the rational allocation of health resources. The medical service suppliers turn from the passive role of controlling expense into active participants. This reduces the workload of medical insurance institution and makes the fee settlement of medical insurance more simple, which saves the cost of management. At the same time, this payment method has obvious shortcomings. The first, it dampens the enthusiasm of medical service personnel $s$ in the case of health care costs have been confirmed. To save medical cost, they reduce the use of new technology, hinder the development of the new medical service technology and influence the treatment effect of patients. Second, because of different productiveness and therapeutic methods for the same disease of different hospitals, it leads that dealing with the same kind of disease has difference. So, it's difficult to calculate the budget of medical treatment insurance accurately for the medical insurance agency. Too high medical treatment budget will produce enormous waste of medical fund. However, a low budget will lead that doctors may shuffle critical patients, and reduce the doctor's work enthusiasm. The doctors unavoidably concerns a lot in the process of treatment, making low medical service quality. Finally, patients are unable to get reasonable medical service. Consequently, the patients' condition will be delayed. [5]

\section{Medical Insurance Payment Pattern in America}

In the United States, the medical insurance payment pattern is the combination of prepaid system and quota settlement. Prospective payment system (PPS) is a kind of method that pay in advance before the cost has happen. This method combines the number of the doctor's income and treatment. In such way, according to the number of attending medical insurance, the number and times of annual reception and cost level of reception in certain area, measure and calculate the total fee of annual compensation control. The handling institutions will prepaid regularly and implement the payment pattern of the total amount control, overall rationing system and share the part over the total fee. This payment will base on the hospital's total expenditure before eliminating unreasonable expenditure according to pay the annual total costs to the hospital. As calculating the annual budget, there are many factors must be taken 
into consider, such as, the hospital scale, the quality of services, population density of service area, death rate, and the hospital is the teaching hospital or not, hospital facilities and equipment conditions, last year's fiscal deficit or surplus situation and inflation. Then confirm the total budget of medical cost next year, and usually change again every one year after negotiating. As you can see, this way of paying for hospital services has a high degree of control. Medical institutions adopting this way must provide services in the scale of health insurance to patients. As a result, medical institutions will be meticulous in the use of the total budget, and control excessive medical services. [6] At the same time, under the total budget system, once the hospital's budget system is established, the hospital's income can't increase with the increase of services. When the loss appears, insurance institutions no longer pay any more, and the loss part will be burden by the hospital. Quota settlement classifies according to the disease diagnosis to control everyone's medical expense within a certain amount. This pattern controls the rising of health care cost effectively.

The form of the combination of prepaid system and quota settlement is quota settlement, capitation payment and Diagnosis Related Groups System. Quota settlement is a payment model of limiting the hospital's income through controlling hospitalization days. Specifically, the cost of hospitalization occupies a large proportion of medical expense, the doctor will be more accurate and reasonable to judge how long will the patient should stay in hospital to control the length of stay. Because if the treatment cost of a patient is beyond the money given by medical institutions, the doctor will afford the excessive part individually. Whereas, the doctor can get commission from the medical institutions. Capitation payment is that calculate the average cost of each applicant. Then each doctor will get the money in base on the patient number. The part over budget shall be borne by the hospital itself, balance part is owned to medical institutions [7].

The diversity of American medical insurance payment method has obvious advantages. Beside the advantages mentioned above, the payment pattern also has the advantages of capitation payment and Diagnosis Related Groups System.[8] The advantage of Capitation payment is simple operation, and don't need high management costs, and easy to control cost. It is helpful to strengthen internal management for the medical service supplier and increase the consciousness of the cost and economic responsibility of the hospital. Meanwhile, it reduces the phenomenon of excessive medical services happened in the hospital, prompt medical service supplier to do the prevention work and reduce the possibility of being ill of the attenders and lessen the medical cost. The advantage of Diagnosis Related Groups System is that the medical service supplier can control the cost of hospitalizing of each patient each time, improve the work efficiency of the hospital, decrease service costs, shorten hospitalization days, and reduce the phenomenon of induced demand. Because the high management requirement of Diagnosis Related Groups System, it will lead the level of management of the medical insurance system to rise and make the management more standardize and scientific.[9]

\section{Countermeasures and Suggestions}

Medical insurance payment under the management of insurance is not perfect, but it still plays an improvement role in many aspects, especially in cost control. So Chinese government can take example by various medical insurance payment pattern in America. The Suggestions were put forward as following.

First, it's to carry out the total prepaid amount. This way is explored actively in our 
country. Some places have become experimental units. The total prepaid system is to set up a top line for medical services within the prescribed medical year. No matter how much the suppliers cost actually, the amount will be the top line. The top line determines the amount the medical insurance management institutions should pay the medical institutions. The rest part is owned to the medical institutions, while the over part will be paid by the medical institutions themselves. Under this system of payment, medical institutions will make reasonable use of medical equipment, and never overbid, which decreases the expenditure of the medical insurance fund.

The second, it's to adopt capitation payment method. Patients will choose to believe the doctor's misleading because they are short of medical knowledge. But if we adopt capitation payment system, that pay the health care cost before treatment. So when policy-holders go to see a doctor, the doctor will carefully consider how to properly treat patients under the condition of limited amount of fee. The doctor will cure the patients with little cost. Because the principle of capitation payment is that the rest belongs to the hospital, the medical institutions themselves needs to be in charge with the excessive part.

Finally, it's to adopt commercial insurance as the leading medical insurance model. We think that the best supervision mechanism is the market competition. America's insurance system is the commercial insurance as the leading, while China's medical insurance is social insurance as a leading. But there are a lot of problems occur both in commercial insurance and social insurance. So we might try to combine the commercial insurance and social insurance. Medical service is not a complete commercial service industry. This decides the importance of social medical insurance. Healthy medical is related with everyone's interests. Health insurance is welfare, public and marketization business equipment. It cannot maximize the welfare of all the people. China can gradually introduce commercial medical insurance and improve its position in the medical insurance mechanism, and weaken the role of social insurance in society. Reform of medical insurance payment pattern is not reached in one step, but a gradual process. It will take plenty of time to verify its applicability and acceptability of each new way. As we have spent a lot of time, payment way of our country's medical insurance also can be various, perfecting medical insurance in China together.

\section{Acknowledgments}

This work is supported by Humanities and Social Sciences Fund of Ministry of Education under grant 12YJC790190, National Social Sciences Fund under grant 13AZD071, National Soft Science Research Project under grant 2013GXS2D020 in China, Young Academic Backbone Support Program under grant 1253G029, Postdoctoral Scientific Research Initiation under grant LBH-Q14094, Soft Science Technology Foundation under grant GC13D101 and QC07C117 in Heilongjiang Province in China.

\section{Reference}

[1] Qi-lin Zhang, The Search on Medical insurance System in America (in Chinese), Beijing: People's Press, 2005, pp.22-24.

[2] Ying Shang, Reform and Analysis on Medical insurance System in America (in Chinese), Jiangsu: People's Press, 2009, pp.267-272. 
[3] Song-ji Li,Yong-fen Gu, Reform and improve medical insurance system in China (in Chinese), Business Studies, 6(2002) 78-84.

[4] Wen-ming Lu, The new plan for publishing medicare in America (in Chinese), Health Protection Management of Jiangsu, 2006, pp.33-36.

[5] Yu Zeng, Reform and Development of Medical Insurance System (in Chinese), Beijing: China Social Press, 2001, pp.56-58.

[6] Dong-yaSun,The role of the commercial health insurance has not been fully played in Chinese medical insurance (in Chinese), Beijing: Medical Insurance in China, 3(2013) 17-18.

[7] Wen Chen, The point of focus of basic medical insurance payment system's reform (in Chinese), China's Health Resources, 2013.

[8] Goldsmith, Association William W, Welfare in America, Journal of the American Planning Spring, 2000, pp.89-92.

[9] Sharon, K. L, Alshadye, Commercial plans in medical managed care: understanding who stays and who leaves, Health affairs, 4(2005) 124-126. 